\title{
Diocotron Wave Echoes in a Pure Electron Plasma
}

\author{
J. H. Yu and C. F. Driscoll
}

\begin{abstract}
Experimental images illustrate spatial Landau damping of an externally launched diocotron mode, and the resulting echo from a second externally launched wave. Filaments at the resonance layer exhibit spiral wind-up during the collisionless damping process, and "unwind" during the echo formation.
\end{abstract}

Index Terms-Diocotron, echo phenomenon, electron plasmas, Kelvin waves, spatial Landau damping, two-dimensional vortex dynamics.

$\mathbf{T}$ HE ECHO phenomenon beautifully exemplifies the reversal of the microscopic phase evolution of a system by a macroscopic operation. Here, the phase evolution is a result of spatial Landau damping. The external excitation of a second wave reverses the phase evolution of this damping, resulting in an echo.

Echoes have been previously observed in longitudinal electron plasma waves [1], but no diocotron wave echoes have previously been seen experimentally. In nonneutral plasmas, diocotron waves are low frequency $\mathbf{E} \times \mathbf{B}$ drift modes representing surface waves or bulk shape distortions of the plasma column. This paper focuses on temporal echoes of diocotron waves in pure electron plasmas, demonstrating the reversible nature of spatial Landau damping. The echo phenomenon has been studied numerically in two-dimensional (2-D) fluid shear flows [2], [3].

We use a cylindrical Penning-Malmberg trap which contains strongly magnetized electron columns to study the echo. In the 2-D $\mathbf{E} \times \mathbf{B}$ drift approximation, the dynamics of single species plasmas is isomorphic to that of 2-D incompressible inviscid fluids. The density $n(r, \theta)$, drift velocity $v(r, \theta)$, and electrostatic potential $\phi(r, \theta)$ of the electron plasma correspond to the vorticity, velocity, and streamfunction of the 2-D ideal fluid, respectively [4].

The main diagnostic is a charge coupled device (CCD) camera, which images a phosphor screen onto which the plasma is dumped. A time evolution, as in Fig. 1, is achieved by dumping essentially identical plasmas at different times, giving $n(r, \theta, t)$. The "perturbation" images displayed are obtained by subtracting the initial $\theta$-symmetric density image from the density image of the plasma with waves, giving $\delta n(r, \theta, t)$.

\footnotetext{
Manuscript received July 2, 2001; revised October 22, 2001. This work was supported by the National Science Foundation under Grant PHY-9 876999 and by the Office of Naval Research under Grant N00014-96-1-0239.

The authors are with the Physics Department, University of California, San Diego, La Jolla, CA 92093 USA (e-mail: fdriscol@ucsd.edu).

Publisher Item Identifier S 0093-3813(02)03301-5.
}

The diocotron waves studied here vary as $\delta n=\delta n_{o}(r)$ $\exp (i m \theta-i \omega t)$. An initial $m_{i}=2$ wave is excited by applying $20 \mathrm{~V}$ to two wall sectors for about one half of an $m_{i}=2$ period. Fig. 2 is an oscilloscope trace of the signal received from two wall sectors separated by $180^{\circ}$. The first pulse represents the initial wave decaying in about five oscillations due to spatial Landau damping [5], [6]. Spatial Landau damping is a collisionless process and requires a resonance between the wave and the particles in the plasma, such that the particles' rotation frequency $\omega_{\boldsymbol{E} \times \boldsymbol{B}}(r)$ equals the wave rotation frequency, i.e., $\omega_{E \times \boldsymbol{B}}=\omega / \mathrm{m}$. The first three images of Fig. 1 show the $m_{i}=2$ wave forming filaments near the resonant radius and wrapping up.

A second $m_{t}=4$ tickler wave is then excited $500 \mu$ s later [Fig. 1(d)] by applying a $20 \mathrm{~V}$ kick to four wall sectors separated azimuthally by $90^{\circ}$. The second pulse of Fig. 2 shows the time at which the $m_{t}=4$ tickler wave is excited. This signal is not the $m=4$ wave oscillation but rather a direct coupling of the tickler excitation to the wall detector.

The tickler wave nonlinearly interacts with the remnants of the initial wave to produce an echo response with $m_{e}=m_{t}-$ $m_{i}=2$. The third wave packet of Fig. 2 is the received echo. The "unwrapping" process can be seen in Figs. 1(f)-(h), and the coherent echo response peaks at the predicted time of $2 \tau$, demonstrating the reversible nature of spatial Landau damping. These may also be considered phase space images, so the filamentary images directly illustrate the theory ideas of phase mixing (and unmixing).

Theory [7] predicts the time of the echo to be $t_{e}=\tau m_{t} /\left(m_{t}-m_{i}\right)$, where $\tau=t_{t}-t_{i}$. Fig. 2 shows agreement with this expression. No echo is seen if $m_{t}<m_{i}$. For small kick voltages, the echo amplitude increases linearly with applied kick voltages.

The detection of the echo in a pure electron plasma is striking, since nonideal effects may break the analogy to 2-D ideal fluids. For example, strong collisional viscosity would destroy any memory of the initial wave in the density distribution. In addition, the confining fields at the ends of the trap influence the bounce averaged $\mathbf{E} \times \mathbf{B}$ drift of the electrons by producing additional $\theta$-drifts. Moreover, particles with different axial energies penetrate a different depth into the end sheaths, also affecting the bounce averaged $\mathbf{E} \times \mathbf{B}$ drift. Thus, the echo may prove to be an incisive tool to characterize these nonideal effects. 


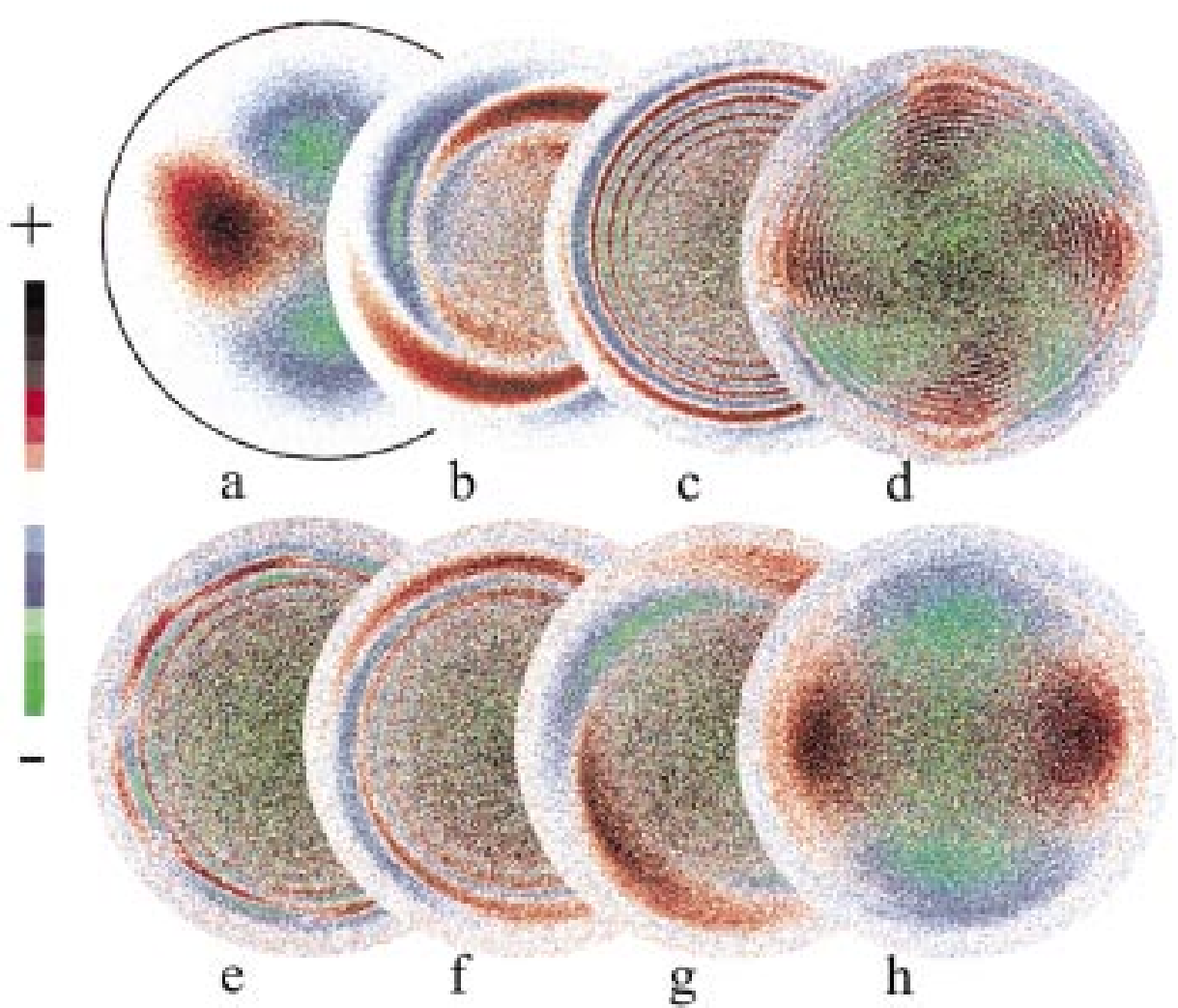

Fig. 1. These images are experimental density perturbations at eight successive times. The color scale is arbitrary. (a)-(c) represent the $m_{i}=2$ initial perturbation and spiral wind-up due to spatial Landau damping. (d)-(e) show the externally launched $m_{t}=4$ tickler wave and subsequent damping. Remnants of the $m_{i}=2$ wave can be seen as thin filaments. (f)-(h) depict the echo formation due to the tickler wave nonlinearly interacting with the initial wave, and the echo amplitude peaks in (h). The black circle in (a) shows the trap wall at $R_{w}=3.5 \mathrm{~cm}$.

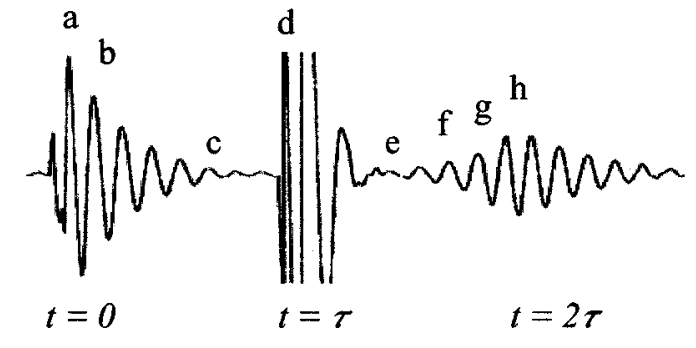

Fig. 2. Oscilloscope trace of the signal received from two sectors of a wall cylinder. The two externally excited waves and resulting echo can be seen. The letters correspond to the time of each density perturbation in Fig. 1.

\section{REFERENCES}

[1] J. H. Malmberg, C. B. Wharton, R. W. Gould, and T. M. O'Neil, "Observation of plasma wave echoes," Phys. Fluids, vol. 11, pp. 1147-1153, 1968.

[2] J. Vanneste, P. J. Morrison, and T. Warn, "Strong echo effect and nonlinear transient growth in shear flows," Phys. Fluids, vol. 10, pp. 1398-1404, 1998

[3] D. A. Bachman, "Nonlinear Phenomena in a Pure Electron Plasma Studied with a 2-D Fluid Code," Ph.D. dissertation, California Institute of Technology, Pasadena, CA, 1997.

[4] C. F. Driscoll and K. S. Fine, "Experiments on vortex dynamics in pure electron plasmas," Phys. Fluids B, vol. 2, pp. 1359-1366, 1990.

[5] R. J. Briggs, J. D. Daugherty, and R. H. Levy, "Role of Landau damping in crossed field electron beams and inviscid shear flow," Phys. Fluids, vol. 13, pp. 421-432, 1970.

[6] D. A. Bachman and R. W. Gould, "Landau-like damping in rotating pure electron plasmas," IEEE Trans. Plasma Sci., vol. 24, pp. 14-15, Feb. 1996.

[7] R. W. Gould, T. M. O'Neil, and J. H. Malmberg, "Plasma wave echo," Phys. Rev. Lett., vol. 19, pp. 219-222, 1967. 